\title{
Gouvernement, économie et biopolitique
}

Government, Economy and Biopolitics

\section{Michael Dillon}

\section{(2) OpenEdition}

\section{Journals}

\section{Édition électronique}

URL : http://journals.openedition.org/conflits/17955

DOI : $10.4000 /$ conflits. 17955

ISSN : $1777-5345$

Éditeur :

CCLS - Centre d'études sur les conflits lilberté et sécurité, L'Harmattan

\section{Édition imprimée}

Date de publication : 15 décembre 2010

Pagination : 11-37

ISBN : 978-2-296-54483-3

ISSN : 1157-996X

Référence électronique

Michael Dillon, «Gouvernement, économie et biopolitique », Cultures \& Conflits [En ligne], 78 | été 2010, mis en ligne le 06 mars 2012, consulté le 30 mars 2021. URL : http://journals.openedition.org/conflits/ 17955 ; DOI : https://doi.org/10.4000/conflits. 17955 


\section{Gouvernement, économie et biopolitique 1}

\section{Michael DILLON}

Michael Dillon est professeur au sein du département Politics, Philosophy and Religion de l'Université de Lancaster au Royaume Uni. Ses recherches s'intéressent aux questions de sécurité et à la guerre depuis une perspective dérivée de la philosophie continentale. Plus spécifiquement, il s'efforce de comprendre ce qu'il advient de la sécurité lorsque les discours de la sécurité et les technologies mobilisées à ces fins prennent pour objet la vie plutôt que la souveraineté territoriale. Michael Dillon est l'auteur de Politics of Security: Towards a Political Philosophy of Continental Thought (Routledge, 1996) ainsi que de nombreux articles sur la sécurité, la guerre, la théorie politique de l'international et la philosophie continentale. Parmi ses plus récentes publications, on compte notamment : Biopolitics of Security in the 21st Century: A Political Analytics of Finitude, Routledge, 2010 ; Deconstructing International Politics, Routledge 2010; The Liberal Way of War: Killing to Make Life Live, Routledge, 2009 (avec Julian Reid) ; Foucault on Politics, Security and War, Palgrave/Macmillan, 2008 (avec Andrew Neal); "Underwriting Security”, Security Dialogue, Vol. 39, 2/4, 2008 ; "Biopolitics of Security in the 21st Century: An Introduction”, Review of International Studies, Vol. 34, No. 2, April 2008. (avec Luis Lobo-Guerrero); "Governing Terror: The State of Emergency of Biopolitical Emergence”, International Political Sociology, Vol.1, No.1, 2007 ; "Biopolitics of Security: Foucault and Agamben", in Peter J. Burgess, ed., New Security Studies, Routledge, 2010.

$$
\begin{aligned}
& \text { "... la fin du gouvernement est dans les choses qu'il } \\
& \text { dirige... "2 }
\end{aligned}
$$

1. Une version reprise et modifiée de cet article sera publiée dans le prochain ouvrage de l'auteur, Biopolitics of Security in the 21st century, à paraitre chez Routledge en 2011. La traduction a été coordonnée par Audrey Kiéfer et David Risse, réalisée par Alexandre MacMillan, Luca Paltrinieri et Stéphanie Martens, et enfin, revue et finalisée par Philippe Bonditti et Grégory Salle.

2. Foucault M., Sécurité, territoire, population. Cours au Collège de France 1977-1978, Paris, Gallimard/Seuil, 2004, p. 103. 
$\mathrm{P}$ our ceux qui nourrissaient l'espoir de comprendre les propositions de Michel Foucault à propos de la naissance de la biopolitique, le cours du même nom qu'il donna au Collège de France en 1978-1979 sonne comme une déception. Il s'agit du dernier cours d'une série de trois. Le premier avait été $I l$ faut défendre la société ${ }^{3}$, en 1975-1976 ; le second Sécurité, territoire et population en 1977-1978 4 . Foucault y avait abordé la formulation du politique et de la sécurité à l'ère moderne en général, de la gouvernance (governance) ${ }^{5}$, du biopouvoir et de la biopolitique en particulier. Foucault avait anticipé cette déception que risquait de susciter à ses yeux La Naissance de la biopolitique ${ }^{6}$, s'excusant même, en ouverture de la huitième Leçon, de s'être détourné du plan annoncé du cours. "Je voudrais vous assurer, malgré tout, que j'avais bien l'intention, au départ, de vous parler de biopolitique et puis, les choses étant ce qu'elles sont, voilà que j'en suis arrivé à vous parler longuement, et trop longuement peut-être, du néo-libéralisme, et encore du néolibéralisme sous sa forme allemande $» 7$.

Naissance de la biopolitique est une interrogation soutenue sur les transformations fondamentales, théoriques et institutionnelles de l'économie à la fin des années 1930 et 1940, en Allemagne en particulier, mais aussi sur la reformulation de la catégorie de «l'économie » en tant que telle et la reconfiguration de la relation entre l'Etat et l'économie. Comme Foucault l'avait luimême noté à propos de l'influente école de théorie économique de Fribourg en particulier : "L'école de Fribourg n'a pas simplement développé une théorie économique, ni même une doctrine. Elle a repensé tout le rapport économie et politique, tout l'art de gouverner. Et pour une bonne raison, c'est qu'elle a eu à se colleter avec un phénomène historique considérable » 8 . Ce phénomène, c'était bien sûr la problématique politique liée au rétablissement du gouvernement en Allemagne et à la fondation d'un nouvel Etat allemand après les catastrophes successives des trois régimes précédents : l'Empire, la République de Weimar, et le Troisième Reich. Cela ne renvoyait pas seulement au contexte propre à l'expérience allemande de la crise du capitalisme

3. Foucault M., Il faut défendre la société. Cours an Collège de France 1976, Paris, Gallimard/Seuil, 1997. Dans la version originale de ce texte, l'auteur renvoie aux versions traduites en anglais : Society Must be Defended, New York, Picador, 2003 ; Security, Territory, Population, Londres, Palgrave Macmillan, 2007 ; The Birth of Biopolitics, Londres, Palgrave Macmillan, 2008 ; The Order of Things, Londres, Palgrave Macmillan, 1969. Dans cette traduction, nous portons les dates des premières publications en français.

4. Foucault M., Sécurité, territoire, population, op.cit.

5. Nous rendrons ici governance par « gouvernance » pour coller au texte original. Cette traduction littérale est toutefois source de confusion conceptuelle, dans la mesure où il ne s'agit pas d'un terme foucaldien : on ne saurait confondre, en français, « gouvernance » et " gouvernementalité ». C'est là l'un des problèmes de traduction posés par le double transfert transatlantique des catégories et concepts [NDLR].

6. Foucault M., Naissance de la biopolitique. Cours au Collège de France 1978-1979, Paris, Gallimard/Seuil, 2004.

7. Ibid., p. 191.

8. Ibid., p. 96. [Il s'agit de notes de manuscrit ajoutées par les éditeurs, mais non lues par Foucault - NDLR] 
symbolisée par l'hyperinflation des années 1920 et la grande dépression des années 1930, mais aussi à celui de la crise théologico-politique précipitée par la chute de la synthèse culturelle-protestante (cultural-protestant) de la période Wilhelmienne, notamment évoquée dans les travaux des juristes Schmitt, Kelsen, et Petersen, des théologiens Buber et Barth et des influents penseurs juifs, de Rosenzweig à Benjamin. Foucault ne tient pas compte de cet arrière-plan aux développements qu'il explore, et reste principalement préoccupé par les trois lignes argumentatives qui suivent.

Il développe d'abord la manière dont la crise du capitalisme était en même temps une «crise de gouvernance »; plus particulièrement une crise de la formulation même de la gouvernance en tant que telle, et pas seulement la crise d'une révision de classe des instruments d'Etat, bien que de tels développements aient aussi été clairement en jeu. Par opposition à la réception de ses travaux par une grande partie du monde académique anglo-saxon, gouvernement et économie sont liés dès le départ dans les analyses de Foucault sur les relations de pouvoir modernes. Ils sont tous deux enfermés dans une appartenance historiquement émergente et mutuellement révélatrice qui semble ellemême dépendre, selon Foucault lui-même, d'une grande variété de pratiques de distinction non moins historiques, parmi lesquelles celles ayant conduit à différencier l'Etat de la société et l'Etat de l'économie - et devenues fondatrices de la modernité. S'il avait laissé de côté le rôle de l'économie dans son œuvre maîtresse Les Mots et les choses ${ }^{9}$, l'intérêt qui est le sien dans ces cours réside bien dans la manière dont l'Ecole de Fribourg, par exemple, a traité le gouvernement et l'économie ensemble, d'un même geste. J'insiste dès lors sur le fait qu'il n'y avait là rien de nouveau pour Foucault. Il avait déjà clairement montré dans ses cours l'année précédente (Sécurité, territoire, population) combien, par exemple, là encore, l'économie et la gouvernance étaient redevables l'une de l'autre dans l'affirmation de la problématique du gouvernement entre le XVI et le XVIII siècle ; en fait, également, dans la différenciation de ce que nous appelons désormais "l'économie ». Eu égard à la biopolitique, j'ajouterais également, et par-delà même Foucault, que la réduction de la vie à ses propriétés biologiques aggrave cette «économisation » (economising) de l'existence, car elle universalise le caractère utilitaire qui découle de la réduction de la «vie » à la vie biologique. Même si sa survie au travers des transactions avec son milieu est décrite. Même si la complexité de ses adaptations et de ces changements, d'après les orthodoxies contemporaines, est prise en compte. Tout comme la vie biologique n'est rien sinon la vie déterminée par l'utilité, l'économie moderne n'est rien sinon la science de l'utilité.

Ensuite, Foucault examine la façon dont la crise de l'économie et de la gouvernance a conduit, en Allemagne - et plus largement, selon moi, à travers toutes les sociétés du bassin de l'Atlantique Nord et leurs ramifications impé-

9. Foucault M., Les Mots et les choses, Paris, Gallimard, 1966. 
riales dans le monde - à l'introduction d'une "politique de l'économie » (“economic polity"). Je distinguerai plus tard l'économie politique ("politique économique") de la politique de l'économie ("economic polity"). Il suffit de dire ici que l'économie politique présuppose ce que j'appellerais une constitution composite d'ontologies régionales - celles, en l'espèce, de l'Etat et de l'économie. En leur sein, et sus d'autres considérations politiques, la légitimité politique s'est fondée sur la manière dont le gouvernement de l'Etat était jugé, d'une part relativement à sa capacité à autoriser "l'économie » à opérer en fonction de ses propres principes, et d'autre part et plus généralement, eu égard à sa capacité à gouverner en fonction d'une large variété d'objectifs déterminés : ce que Foucault appelait des «finalités spécifiques »10. Dans le processus de transformation du gouvernement et de l'économie intervenu au cours de la première moitié du xxe siècle, en Allemagne et dans la Communauté économique européenne en particulier, la politique et l'Etat - et plus généralement les aspirations à une union politique - se sont d'abord fondés sur l'établissement et le fonctionnement ordonné d'économies de marché prospères.

Quoique certains jugent cette autonomie factice, l'économie politique a prétendu que la politique et l'économie relevaient d'ontologies régionales d'existence indépendantes. Dans l'établissement de «politiques de l'économie » (economic polities), cependant, le domaine politique dérive des dynamiques fructueuses de l'économie de marché - un quasi épiphénomène pourrait-on même dire. Pour Foucault, il s'agit là moins du résultat d'une structure de classe et d'intérêts de classe que d'une dynamique d'élaboration de la problématique même de gouvernement, et de sa résolution. Le gouvernement, pour Foucault, en est venu à opérer fondamentalement au travers de l'économie ainsi qu'à être jugé principalement eu égard à sa capacité à gouverner en rapport avec elle. Toutefois, ce qui s'est amorcé sous la forme d'une profonde différenciation entre l'Etat et l'économie dans les régimes de pouvoir libéraux - dès lors que le gouvernement de l'Etat libéral des premières heures de la modernité a été pensé dans les termes de ce que Foucault appelle les questions «royales » du pouvoir - est progressivement devenu un amalgame des deux au fur et à mesure que la logique utilitariste de la biopolitique se déployait par gouvernementalisation et biopolitisation complète de l'Etat libéral qui, pour le dire de manière concise, est devenu de plus en plus préoccupé par la gouvernance et la promotion de la vie entendue comme l'existence de l'espèce, elle-même regroupée en cohortes contingentes d'individus rassemblées sous le vocable générique de «population ».

Il va sans dire que ce n'est pas là le seul moyen par lequel la problématique gouvernement/politique/liberté peut être configurée. Pas plus d'ailleurs que la finitude des «finalités spécifiques » auxquelles Foucault fait référence de 
manière si intuitive n'est le seul moyen par lequel l'analytique de la finitude qui gouverne la modernité politique peut être configurée. Je reviendrai à ces termes centraux plus loin. Mais, là encore, retenons que l'analytique biopolitique de la finitude qui émerge historiquement dans le bassin de l'Atlantique Nord n'est pas seulement un phénomène divers et évolutif. Sa réduction de la vie à la finitude de l'existence de l'espèce et la traduction particulière de la série finitude-facticité-liberté sur laquelle repose politiquement cette conception de l'existence de l'espèce ont toujours été sujettes à plusieurs interprétations : principalement - et dans l'objectif de remettre en question la conception même proposée par Foucault dans Les Mots et les choses - entre Kant et Heidegger ${ }^{11}$, ainsi qu'entre Kant, Heidegger et Merleau-Ponty où la question de la "corporéité », ou du corps vivant, est soulevée ${ }^{12}$. Contester la biopolitique de la sécurité au XXI siècle revient dès lors non seulement à contester la réduction de la vie à l'existence de l'espèce mais aussi à contester l'analytique de la finitude, de la facticité et de la liberté qui opérationnalise cette réduction de la vie mortelle à ce qui est évidemment une forme profondément aporétique et de plus en plus autodestructrice d'existence biopolitique, localement et globalement.

Si je partage avec Foucault l'idée que notre politique de sécurité moderne - qui oscille constamment entre l'empirique et le transcendantal, ou ce que je dépeindrais plutôt comme l'empirique et l'eschatologique -, fournit une expression de l'aporie de l'analytique de la finitude, je m'accorde avec Béatrice Han ${ }^{13}$ pour affirmer que Foucault tombe en quelque sorte sous le coup de sa propre critique de cette condition aporétique. Ayant renversé le sujet kantien qui fait l'histoire en observant la manière dont l'histoire elle-même produit ce sujet, Foucault n'en retourne pas moins à ce même sujet pour faire valoir en permanence la capacité - critique - de ce dernier à refaire l'histoire par recours à l'a priori historique qui n'est autre, bien sûr, que sa propre version du transcendantal.

Ici surgit ma différence fondamentale avec Foucault qui suppose une réévaluation significative du fonctionnement de l'analytique de la finitude. Oui, tant que la biopolitique moderne de la sécurité est une expression du couple savoir/pouvoir profondément influencé par des conceptions changeantes des propriétés biologiques des humains et du caractère de l'existence

11. Heidegger M., Phenomenological Interpretations of Kant's Critique of Pure Reason, Bloomington, Indiana University Press, 1997; Rafoul F., Nelson E. S. (eds.), Rethinking Facticity, Albany, State University of New York Press, 2008 ; Schalow F., The Renewal of the Heidegger-Kant Dialogue, Albany, State University of New York Press, 1992 ; Stambaugh J., The Finitude of Being, Albany, State University of New York Press, 1992.

12. Flynn B., Political Philosophy at the Closure of Metaphysics, Atlantic Highlands, N.J., Humanities Press, 1992, et du même auteur, "Merleau-Ponty on Fact and Essence”, in Rafoul F., Nelson E. S. (eds.), Rethinking Facticity, op.cit. ; Merleau-Ponty M., The Visible and the Invisible, Chicago, Northwestern University Press, 1968.

13. Han B., Foucault's Critical Project. Between the Transcendental and the Historical, Stanford, Stanford University Press, 1998. 
de l'espèce en général, les biopolitiques de la sécurité du XXe siècle restent également profondément enserrées dans l'impasse aporétique de l'analytique kantienne de la finitude - dont elles sont d'ailleurs une expression politique d'un point de vue épistémologique. Si l'analytique kantienne de la finitude nécessitait la figure anthropologique de l'Homme pour déployer son projet, le référent épistémologique et empirique moderne de la biopolitique contemporaine est moins l'Homme que l'espèce. La biopolitique est dès lors moins une humanisation dérivée de la machine anthropologique de l'Homme qu'une spéciation impitoyable dérivée de la machine techno-instrumentale biopouvoir/savoir.

Toutefois, l'analytique kantienne de la finitude n'est pas la seule expression de la finitude. Nous ne pouvons ici faire l'économie du débat HeideggerKant (Heidegger's auseinandersetzung with Kant, dans le texte original) précisément sur ce point :

«Le mouvement qu'opère Hegel, depuis Kant jusqu'à l'idéalisme absolu, est l'unique cohérence du développement de la philosophie occidentale. Ce développement est possible et nécessaire à travers Kant car le problème du Dasein humain, la finitude, n'est pas devenu un véritable problème pour lui et donc pas un problème central de la philosophie car Kant lui-même - comme le montre la seconde édition de la Critique de la raison pure - s'efforça de dépasser une finitude pleine d'incompréhensions pour trouver son apaisement dans l'infinité 14 »

L'analytique kantienne de la finitude, telle qu'en rend compte Foucault dans Les Mots et les choses, n'en est pas moins devenue un point de départ fondateur pour la sécurité à la fois de la gouvernance et du savoir, en fait pour assurer la gouvernance par le savoir. Parce que l'épistémologie moderne n'est pas seulement un projet de sécurité fondé sur le sujet moderne doué de savoir, la formation continue d'un tel sujet, individuellement et collectivement, est de plus en plus devenue le projet politique moderne après que la métaphysique des Lumières des années 1780 eut insisté, à travers Kant, sur la nécessité qu’il y avait à faire d'un tel sujet l'imaginaire politique même de la modernité ${ }^{15}$.

Cependant, Foucault interprète le pouvoir en terme non-subjectiviste. Comme pour servir sa révision de la critique, il s'efforce continuellement de sauver le sujet dans son exercice d'historicisation de ce dernier 16. Ainsi n'y at-il pas, même chez Foucault, de politique moderne sans sujet moderne. De la même manière, cependant, il n'y a pas de sujet moderne sans le travail gouver-

14. Cité dans Stambaugh J., The Finitude of Being, op.cit., p. 3.

15. Campbell D., Dillon M. (eds.), The Political Subject of Violence, Manchester, Manchester University Press, 1993.

16. Han B., Foucault's Critical Project, op.cit. 
nemental sisyphéen qu'est la politique moderne ${ }^{17}$. Tel n'est pas le cas avec Heidegger qui échappe à l'aporie de l'analytique moderne de la finitude, seulement, bien sûr, pour souscrire à une autre. Cela dit, il nous faut aussi considérer jusqu'à quel point la dissolution du sujet a pu aller ces dix dernières années en réponse non aux analyses foucaldiennes mais aux développements techno-scientifiques considérables - et pas seulement ceux liés aux révolutions numériques et moléculaires, mais aussi ceux liés aux neurosciences, par exemple. Tout observateur perspicace, sachant compter et doté d'un minimum de connaissances scientifiques aura pu noter :

«Il y avait, il n’y a encore pas si longtemps, une opposition absolue entre le soi et autrui : un 'je’ identique à lui-même, totalement présent en tant que psyché intérieure, autonome et indivisible, opposé à une collectivité extérieure et amorphe de tierces personnes. Aujourd'hui, l'unité je/moi se dissout. Celui ou celle qui dit ou écrit 'je' n'est plus une totalité singulière, mais multiple : une pluralité mouvante de 'parties-je', 'rôles-je', 'fonctions-je' et 'présences-je'. Le 'je' se disperse dans le collectif, et le collectif s'immisce, s'insinue et s'internalise au sein du moi. Ce qui était propre à l'individu et constituait son for intérieur est envahi par le public, l'historique, le social. Ce qui constituait le monde extérieur des événements pénètre (comme il semble l'avoir toujours fait) l'âme de l'individu sous le couvert du destin personnel. 18 »

Il n'est cependant pas nécessaire de souscrire à la conception heideggérienne de la finitude - bien que, dans une certaine mesure, ce soit le cas pour moi - pour constater qu'elle diffère de celle de Kant, et dans quelle mesure. Ce contraste peut constituer le point de départ non seulement d'une critique de la description foucaldienne de l'analytique de la finitude moderne formulée en termes kantiens, mais également des profondes ambivalences qui accompagnent la tentative de Foucault d'y échapper ${ }^{19}$. Ma lecture de Foucault s'en éloigne selon deux axes principaux.

Le premier est simplement d'ordre historique. Il concerne le changement de statut de la vie de l'espèce depuis le croisement des révolutions numérique et moléculaire au XXe siècle, et leur intégration massive à une biopolitique de la sécurité qui caractérise les sociétés du bassin de l'Atlantique Nord. Une biopolitisation de la sécurité qui se manifeste à travers une guerre infinie contre le terrorisme, et d'une manière plus générale à travers l'ubiquité de la surveillance et de la sécurité qui caractérise aujourd'hui l'économie de la gouver-

17. Campbell D., Dillon M. (eds.), The Political Subject of Violence, op.cit.

18. Rotman B., Becoming Beside Ourselves: The Alphabet, Ghosts, and Distributed Human Being, Durham and London, Duke University Press, 2008, p. 99. En gras dans le texte original de cet article.

19. Han B., Foucault's Critical Project, op.cit. 
nance biopolitique tout court ${ }^{20}$. Ici, d'une certaine façon, je prolonge le projet de Foucault consistant à étudier les mutations historiques des rationalités biopolitiques et des technologies de gouvernement du biopouvoir en rendant compte de la manière dont elles sont devenues plus intenses et extensives dans la première décennie du $\mathrm{XX}^{\mathrm{e}}$ siècle. J'affirme que la biopolitique de la sécurité au XXIe siècle prend une dimension hyperbolique, dans la mesure où en construisant la vie comme une entité en émergence constante, elle institue la gouvernance de la vie comme une urgence constante. Il s'agit cependant ici d'une urgence de l'émergence qui diffère à la fois des travaux de Schmitt et de ceux de Benjamin qui ont grandement influencé les débats actuels sur ce sujet ${ }^{21}$.

Le second est d'ordre théorique et analytique. Il touche au cœur même du projet foucaldien et au projet critique de la modernité. Sur ce point, je me distancie de Foucault en affirmant que la modernité - en particulier ses rationalités biopolitiques et ses technologies de gouvernement - est fondée sur, et influencée par la pensée de la finitude. Une pensée de la finitude qui, depuis Machiavel et d'un point de vue politique, a envahi le champ génératif de formation de la politique moderne ; c'est-à-dire bien avant la révolution copernicienne de Kant et son analytique de la finitude que, d'un point de vue épistémologique, Foucault a qualifié de seuil de l'épistémè moderne - seuil dont la chute allait annoncer la dissolution de la figure anthropologique de « l'Homme » sur laquelle, de manière brève et sans succès, cette même épistémè s'était appuyée. Le diptyque empirico-transcendental ne caractérise pas uniquement la modernité kantienne. Sous la forme empirico-eschatologique, il a caractérisé la modernité politique depuis les premières tentatives de fonder le politique dans une réalité distincte de celle de la promesse de la vie éternelle ayant servi à établir la distinction entre le message chrétien et le pouvoir pastoral (pouvoir que l'Eglise a cherché à exercer sur le troupeau constitutif de sa croyance œcuménique). Comme l'affirme Jacob Taubes, «l'ontologie, même celle de la rédemption, mobilise et présuppose le corps du Christ comme objet commun $22 »$.

Une rupture historique d'un genre nouveau prend alors place au moment même où la gouvernance et la politique de sécurité en Occident glissent du message sotériologique chrétien au message moderne. Selon moi, ce déplacement correspond à l'abandon du champ de formation politique et gouvernemental centré autour de la pensée chrétienne de la rédemption et de l'éternité. En niant le point de référence qui soutenait la pensée sotériologique de l'éter-

20. En français dans le texte.

21. Schmitt C., The Concept of the Political, Chicago, Chicago University Press, 2007, et Schmitt C., Political Theology II, London, Polity Press, 2008 ; Benjamin W., "Critique of Violence", in Bullock M., Jennings M. J. (eds.), Walter Benjamin Selected Writings, Volume 1, 19131926, Cambridge, Mass., Harvard University, The Belknap Press, 2004, pp. 236-252.

22. Taubes J., The Political Theology of Paul, Stanford, Stanford University Press, 2004, p. 56. 
nel, et en cherchant une multiplicité de points de référence alternatifs à ceux qui avaient structuré les rationalités politiques et les technologies de gouvernement de l'Eglise et de la Cour, la modernité a émergé comme un champ de formation politique centré autour de la pensée de la finitude. C'est pourquoi, si la politique sotériologique de l'ère chrétienne était une politique de l'éternel, la politique de la sécurité de l'ère moderne est plutôt une politique d'une infinité de finitudes temporelles. Certes, la politique de la sécurité moderne est devenue toujours plus épistémologique avec Kant, toutefois préoccupée par la recherche d'un ancrage dans un champ fini, et ce bien avant que Kant ait fait de la finitude le fondement du « connaître ce que nous connaissons »; et elle a toujours cherché à asseoir la connaissance dans le schéma transcendantal qui visait à compenser métaphysiquement les déficiences rationnelles, théologicophilosophiques, de l'ère chrétienne. Cependant, en réintroduisant le transcendantal, Kant avait pris parti dans la longue dispute théologico-politique qui remontait à Eusebius et Saint Augustin dans la tradition chrétienne ${ }^{23}$. Contrairement à d'autres, notoirement Saint Augustin ${ }^{24}$, Kant prit position aux côtés de ceux qui osaient promettre que le message chrétien de la sécurité de la paix éternelle était un projet qui pouvait être réalisé, au moins dans une certaine mesure, ici sur terre ${ }^{25}$. Ainsi les entreprises politiques et philosophiques des XVII et XVIII e siècles ont-elles aidé à ouvrir la voie pour une immanence de l'eschaton si caractéristique de la techno-science contemporaine en général, et de sa biopolitique de la sécurité en particulier.

Troisièmement, Foucault a également cherché à analyser la façon dont les arrangements dans l'Allemagne d'après 1945 - dont les négociations ont déterminé l'ordre d'après-guerre - ont établi de nouveaux « champs d'adversité » composés de nombreuses «surfaces de friction ». "Crises de gouvernance », « champs d'adversité » et « surfaces de friction » sont des termes foucaldiens importants mais négligés. En infléchissant de manière significative l'analyse de Foucault sur le fonctionnement des relations de pouvoir moderne d'une façon générale, et de la biopolitique en particulier, ils dressent un autre portrait des dynamiques des relations de pouvoir contemporaines. En particulier, ils mettent en évidence la manière dont, pour Foucault, les relations de pouvoir matérialisent leurs insuffisances contingentes. Composés d'une grande variété de conflits immanents, ces termes contribuent à expliquer pourquoi et comment les relations de pouvoir se trompent de cible, sont sujettes à des erreurs de calcul, et sont traversées par des résistances pragma-

23. O'Donovan O., The Desire of the Nations? Rediscovering the Roots of Political Theology, Cambridge, Cambridge University Press, 1996 ; O’Donovan O., Lockwood O’Donovan J. L. (eds.), From Irenaeus to Grotius: A Sourcebook in Christian Political Thought 100 - 1625, Grand Rapids, Eerdmans, 1999.

24. Markus R. A., Saeculum: History and Society in the Theology of St. Augustine, Cambridge, Cambridge University Press, 1988.

25. de Vries H., "Introduction”, in de Vries H., Sullivan L. E. (eds.), Political Theologies, New York, Fordham University Press, 2006 ; Pranger M. B., "Politics and Finitude. The Temporal Status of Augustine's Civitas Permixta”, in de Vries H., Sulliva L. E. (eds.), ibid., pp. 113-121. 
tiques et de principe. Si pour Foucault le pouvoir est une toile, il s'agit d'une toile déchirée. S'il s'agit d'un champ de formation, cette formation est toujours déformante. Si c'est une matrice d'intelligibilité, cette intelligibilité n'est pas seulement l'objet de luttes permanentes - le travail de véridiction, ou «faire le vrai » comme le dit Foucault -, elle est toujours contestée aussi bien de l'intérieur que de l'extérieur. Perméables à tout un ensemble de processus différents, les technologies de pouvoir sont sans cesse modifiées par l'action de nombreux facteurs. Cela signifie « qu'il est tout à fait possible d'atteindre des effets globaux, non pas par des affrontements concertés, mais aussi bien par des attaques locales, latérales ou diagonales qui mettent en jeu l'économie générale de l'ensemble » 26 .

En s'excusant d'avoir eu à consacrer tout un cours sur la biopolitique aux transformations des catégories économiques au milieu du XXe siècle, Foucault obscurcit, malgré lui, les caractères essentiels des rapports entre biopolitique et économie. Cette excuse porte l'auditeur/lecteur à croire qu'en traitant de la révision de l'économie, Foucault n'aborde pas la biopolitique. Pourtant, il le fait. Le problème est qu'il n'explique ni comment ni pourquoi. Ayant auparavant suggéré et détaillé la manière dont la biopolitique contribue à intégrer l'économie au cœur même de la problématique de la politique et du gouvernement moderne - l'objet du cours de l'année précédente -, dans son cours Naissance de la biopolitique, il néglige une explication détaillée du caractère essentiel de la relation entre la biopolitique et l'économie pour insister sur les réponses apportées à la double crise du gouvernement et du capital dans l'Allemagne du milieu du XX siècle.

Foucault a laissé entendre qu'il s'était éloigné de ce thème bien que ce ne fût pas le cas. Cependant, il n’a pas réussi à établir les liens nécessaires entre, d'une part, ses recherches historiques précédentes portant sur les problématisations modernes de la gouvernementalité, de la politique et de l'économie, et d'autre part ses travaux plus récents. Cette insuffisance s'avère aussi théorique qu'historique. L'exploration de la biopolitique initiée dans Sécurité, territoire et population prend fin au début du XIX ${ }^{\mathrm{e}}$ siècle. Foucault ne rend compte de ce qui se produit ensuite que lorsqu'il aborde la crise de l'économie et de la gouvernance à laquelle ont tâché de répondre les Ordoliberalen allemands de la première moitié $\mathrm{du} \mathrm{XX}^{\mathrm{e}}$ siècle, et dont s'est occupée par la suite l'école de Chicago - les anarcho-capitalistes américains comme les nomme Foucault. Comme il l'admet dans ses cours, il ne rapporte pas suffisamment ce travail à l'élaboration de son analytique de la biopolitique.

Bien que nous soyons redevables à Foucault d'avoir posé la question fondamentale qui renseigne l'analytique de la biopolitique, il ne nous dit rien de ce qu'il advient des relations de pouvoir lorsqu'elles prennent l'existence de 
l'espèce pour objet, pas plus qu'il ne développe de réflexion théorique sur la consonance essentielle qui a cours entre l'existence de l'espèce et l'existence de l'économie telle que formulée à partir du XVIII e siècle dans le cadre d'une science de l'utilité. Foucault n'aborde pas non plus la manière dont l'utilité qui opère au cœur des récits à propos de la vie économique et de la vie de l'espèce, noue ces deux éléments dans la formation serrée qui caractérise de plus en plus les politiques économiques du capitalisme libéral global et leur gouvernance.

Je ne vais pas ici combler ce vide historique tant ma préoccupation, inspirée par Foucault et en dialogue avec lui plutôt qu'inscrite dans la stricte prolongation de son travail, porte sur la biopolitique de la sécurité telle qu'elle opère en ce début de XXIe siècle. Notons d'abord que lorsque l'influence des théories biologiques et évolutionnistes de la fin de la période victorienne a été davantage prise en considération, elles l'ont presque toujours été du point de vue de la version conflictuelle du darwinisme social, de la lutte pour la survie et de l'élimination de ceux qui sont inadaptés. Mais on retrouve également un autre lien entre la biologie et la théorie socio-politique de l'idéologie libérale britannique au tournant des XIXe et XXe siècles. Généralement attribuée aux travaux de Thomas H. Green, l'incorporation du collectivisme, du communautarisme et de la réforme sociale aux révisions du libéralisme britannique est davantage à mettre au crédit des courants de pensée biologique et évolutionniste. La biologie et l'évolution devaient alors apporter les preuves « empiriques » de la transition d'une éthique libérale vers un certain collectivisme, tout en corroborant historiquement et philosophiquement les présupposés biopolitiques qui avaient, selon Foucault, influencé les rationalités politiques du libéralisme et les technologies de gouvernement depuis les XVII et XVIII siècles ${ }^{27}$. Mon intérêt pour la biopolitique de la sécurité au XXIe siècle renvoie à ce que je considère être une lacune théorique importante chez Foucault. Rappelant qu'il avait déjà démontré, comme cité en épigraphe de ce chapitre, que les formes de pouvoir modernes s'organisent autour des propriétés des objets qu'elles cherchent à diriger, Foucault montre dans ses recherches historiques comment les propriétés biologiques des êtres humains, dans la mesure où elles expriment ou relèvent de l'utilité, sont devenues objets de gouvernance. D'où l'émergence de la biopolitique pour Foucault, au moins pour Agamben 28.

La recherche historique de Foucault concernant l'émergence de la biopolitique aux premières heures de la modernité répond à une question centrale : qu'advient-il du gouvernement et de la politique - et plus généralement des

27. Freeden M., "Biological and Evolutionary Roots of the New Liberalism in England", Political Theory, vol. 4, n ${ }^{\circ}$ 4, pp. 471-490; Dillon M., Reid J., The Liberal Way of War: Killing to Make Life Live, Milton Park, Routledge, 2009.

28. Mills C., The Philosophy of Agamben, Montreal and Kingston, McGill-Queen's University Press, 2008 ; Dillon M., Lobo-Guerrero L., "The Biopolitical Imaginary of Species Being”, Theory, Culture and Society, vol. 26, n 1, 2009, pp. 1-23. 
relations de pouvoir - lorsqu'ils prennent les caractéristiques biologiques des êtres humains pour objet référent du gouvernement et de la politique ? Par nature, cette question se ramifie, car les propriétés biologiques des êtres humains ont autant donné lieu à diverses interprétations scientifiques que la nature de l'espèce a elle-même été sujette à controverse 29 . Dès lors, comment, les discours politiques et de gouvernement ont-ils été influencés par le pouvoir/savoir des « sciences de la vie » qui, ayant émergé au cours du XIXe siècle, se sont développées de manière exponentielle au XXe ? Qu'est-il arrivé, pouvons-nous également nous demander, à cette forme de gouvernement et de politique dont les rationalités politiques et les technologies gouvernementales étaient fondées sur l'infinité de la finitude temporelle de la «vie » finie entendue comme l'existence de l'espèce - lorsque la concrétisation de la «vie » ainsi comprise a elle-même été transformée par les récits techno-scientifiques à propos de ce qu'est être une chose vivante et finie - à la manière de ce qui s'est passé lorsque les révolutions digitale et moléculaire se sont combinées pour révolutionner les «sciences de la vie » au cours du XXe siècle. Plus généralement, et en plus des nombreuses façons dont ces derniers développements ont radicalement transformé les doctrines stratégiques militaires, les concepts opérationnels, l'entrainement à et l'organisation de la voie libérale de la guerre, comment l'objectif explicite de tuer et l'hyperbolisation générique de la sécurité qui caractérise désormais la gouvernance libérale, localement et globalement, ont-ils influé sur la gouvernance économique sécurisée des sociétés libérales ? En plus de cette hyperbolisation de la sécurité et de la surveillance locale et globale des populations qui suscite aujourd'hui tant d'inquiétudes politiques, quelles sont les conséquences et les implications éthiques aussi bien que politiques et gouvernementales du rétrécissement de la portée de l'existence - son horizon de possibilités ainsi que l'expérience corporelle de l'incarnation vécue - aux rationalités politiques et aux technologies de gouvernement d'une infinité de finitudes temporelles prenant la forme de l'existence de l'espèce, de surcroît opérationnalisées par et au travers des rationalités et de technologies gouvernementales de plus en plus numérisées et molécularisées.

Avant d'aborder cette lacune théorique - notamment à partir du chapitre crucial de Les Mots et les choses intitulé "L'homme et ses doubles », où Foucault décrit comment « l'analytique de la finitude » caractérise la structure aporétique de l'épistémè moderne - il me faut revenir sur la manière dont, dans Sécurité, territoire, population, Foucault décrit la problématique du gouvernement telle que nouvellement posée au Xvie siècle. J'examinerai aussi la manière dont il voit cette problématique de gouvernement installer l'écono-

29. Mayr E., Populations, Species, and Evolution, An Abridgment of Animal Species and Evolution, Boston, Mass., Harvard University Press, 1975 ; Mayr E., Provine W. B. (eds.), The Evolutionary Synthesis. Perspectives on the Unification of Biology, Boston, Mass., Harvard University Press, 1998 ; Monod J., Chance and Necessity. An Essay on the Natural Philosophy of Modern Biology, Harmondsworth, Penguin Books, 1997 ; Jacob F., The Logic of Life, Harmondsworth, Penguin Books, 1989 ; Jacob F., The Possible and the Actual, Harmondsworth, Penguin Books, 1989. 
mie au cœur du problème du gouvernement. Enfin, on notera comment, au fil de sa recherche, Foucault décrit l'évolution de la catégorie de l'économie au fur et à mesure de l'évolution de la problématique même de gouvernement au cours des XVII et XVIII e siècles vers sa biopoliticisation croissante. Le point à ne pas oublier, concernant à la fois la catégorie de l'économie et toutes les notions-clé que Foucault mobilise par la suite - telles que contingence, liberté, économie et gouvernement - est que l'« économie »n'est pas un universel. L'économie a une histoire. Dans cette série de cours, on peut voir Foucault suivre la matérialisation historique et évolutive de la catégorie de l'économie en parallèle de la matérialisation historique et évolutive de la catégorie non moins historique de gouvernement qui lui est intimement liée. Plus tard, lorsqu'il traite de l'avènement de la biopolitique et, plus spécifiquement encore, de l'émergence de la population en tant que référent empirique originaire de la gouvernance biopolitique, Foucault le fait aussi en lien avec les catégories de l'aléatoire et du contingent ${ }^{30}$. Du point de vue de cette analyse, gouvernement, économie et contingence sont tous les trois impliqués dans l'émergence de la sécurité en tant que principe de formation sur lequel en sont venues à reposer, dès les premières heures de la modernité, la problématisation non pas seulement du gouvernement et de l'économie, mais aussi celle de la liberté. Même si, nous le verrons, la sécurité doit être interprétée comme un appareil complexe de nouvelles positivités susceptibles de faciliter, améliorer et maximiser une entité finie en constante évolution appelé la "vie ", dont le caractère principal est précisément sa finitude. Ainsi, convient-il de parler non seulement d'une biopolitique de la sécurité, mais aussi d'une analytique biopolitique de la finitude caractérisant ce que je propose d'appeler la «vie et ses doubles ».

\section{Gouvernement, économie, liberté}

«La liberté et la sécurité, le jeu liberté et sécurité, c’est cela qui est au cœur même de cette nouvelle raison gouvernementale dont je vous donnais les caractères généraux. Liberté et sécurité, c'est cela qui va animer de l'intérieur, en quelque sorte, les problèmes de ce que j’appellerai l'économie de pouvoir propre au libéralisme. ${ }^{31}$ »

Foucault consacre les premiers cours de la série Sécurité, territoire et population à l'émergence de la problématisation du gouvernement au cours des premières heures de la modernité. Celle-ci concerne moins les questions de l'art de gouverner et du développement des premières idées modernes de la souveraineté - lesquelles ont pendant longtemps éclipsé cette question - que la réponse devant être apportée à la crise générique du gouvernement entendu comme «conduite des conduites » née de la dissolution progressive du monde

30. Dillon M, "Governing Terror: The State of Emergency of Biopolitical Emergence", International Political Sociology, vol. 1, $\mathrm{n}^{\circ}$ 1, mars 2007.

31. Foucault M., Naissance de la biopolitique, op.cit., p. 67. 
médiéval. Il examine alors toute une variété de traités oubliés - « une littérature immense, une littérature monotone aussi 32 »- «qui se donnent non plus exactement comme conseils au prince, qui ne se donnent pas non plus, encore, comme science de la politique $[\ldots]]^{33}$ ». Cette littérature, qui se fait jour au cours du xvie siècle et se prolonge au XVII et XVIII ${ }^{e}$ siècles, se présente plutôt comme visant à résoudre le problème du « gouvernement " : gouverner un foyer, une famille, un ordre religieux, une province, des enfants et des âmes aussi bien qu'un Etat. Ces pratiques de gouvernement ont été interprétées comme diverses et variées parce que plusieurs catégories d'individus y ont été impliquées et que le gouvernement lui-même cherchait à gouverner différentes sortes de choses. En ce sens, cette littérature a ouvert la perspective de plusieurs formes de gouvernement définies en fonction des propriétés de la chose ou des choses à gouverner, le gouvernement de l'Etat par le prince n'étant qu'une de ces multiples formes de gouvernement.

«Il me semble », dit Foucault, « que tous ces problèmes sont, dans leur intensité et dans leur multiplicité aussi, très caractéristiques du XVIe siècle, et ceci au point de croisement, pour dire les choses très schématiquement, de deux mouvements, de deux processus 34 ». Le premier mouvement touchait à un thème que Foucault a souvent été accusé d'avoir négligé 35 : «le processus, bien sûr, qui, défaisant les structures féodales, est en train d'aménager, de mettre en place les grands Etats territoriaux, administratifs, coloniaux 36 ». Comme le dit Foucault, le deuxième mouvement est différent, bien que lié au premier de façon complexe : c'est celui par lequel, «la Réforme, puis la Contre-Réforme, [avaient remis] en question la manière dont on [voulait] être spirituellement dirigé sur cette terre vers son salut ${ }^{37}$ ».

Les fondations et les co-relations établies de longue date entre l'Eglise et la royauté via des disputes historiques incessantes et des conflits souvent violents - symbolisées par les deux cités d'Augustin, la cité des hommes et la cité de Dieu - avaient en fait été soumises depuis longtemps à cette réévaluation radicale, cette transformation et ce changement qui ont façonné l'âge moderne, bien sûr marqué par les différends qui l'ont précédé. Comme l'a soigneusement noté Louis Dupré, « la déflagration culturelle que nous appelons modernité a eu lieu seulement quand la nouvelle notion humaniste de créativité a formé un mélange explosif avec les conclusions négatives de la théologie nominaliste (vers la fin du Moyen Age). Son choc brisa l'organicisme typique de l'interprétation occidentale du réel 38 »; et notamment, pour ce qui nous intéresse ici plus particulièrement, le réel qui sous-tendait les doctrines du

32. Foucault M., Sécurité, territoire, population, op.cit., p. 93.

33. Ibid., p. 92.

34. Ibid.

35. Stoler A. L., Race and the Education of Desire. Foucault's History of Sexuality and the Colonial Order of Things, Durham, Duke University Press Books, 1995.

36. Foucault M., Sécurité, territoire, population, op.cit., p. 92 - italique de l'auteur.

37. Ibid. 
gouvernement et de la légitimité politique ${ }^{39}$. Dans les termes de Foucault, cela signifie que Dieu ne gouvernait plus le monde « au sens pastoral ». « [Dorénavant,] il règn[ait] souverainement sur le monde à travers des principes $40 »$.

Plus précisément, Dieu ne "sécurisait » plus le monde non plus. Progressivement, c'est à l'homme que cette tâche revient. Sa gouvernance pastorale quotidienne et son organisation politique se trouvant moins déterminées par la promesse sotériologique du salut éternel, le sujet fini devient architecte de la constitution politique et de l'ordre gouvernemental, sans référence à la sécurité de son fondement supposé dans la loi naturelle divine immuable. La quête de l'ordre ne devait plus seulement se faire par soumission au «Dieu mortel » de nouvelles souverainetés émergentes aux XVII et XVIII e siècles, mais aussi au travers des technologies plus larges, de discipline et de gouvernance, que ces souverains - traumatisés par les guerres religieuses du XVII siècle considéraient eux-mêmes comme vitales à l'établissement de la paix nationale et internationale ${ }^{41}$.

Vers la fin du Moyen Age, la théologie nominaliste avait écarté Dieu de la création de manière quasi définitive ${ }^{42}$. Le divin était de plus en plus relégué dans une sphère supranaturelle distincte de la nature avec laquelle elle ne conservait désormais plus qu'un lien de causalité. Alors qu'auparavant le sens et la signification des choses étaient établis dans l'acte même de la création par un Dieu infiniment sage et providentiel, c'est à l'esprit humain qu'il revint d'interpréter un cosmos dont la structure n'était plus tant donnée comme intelligible à l'homme que posée par lui. Au lieu d'être partie intégrante d'un infini cosmologique, un sujet humain fini émergea, chargé de formuler sa vérité et de postuler son sens, dont le fondement devenait dès lors moins le mystère de l'infinité divine que la positivité de la finitude humaine. Alors que, d'un point de vue onto-théologique, le réel se divise en deux sphères - celle de l'esprit qui contient toutes les déterminations intellectuelles, et celle de tous les autres êtres qui les reçoivent - le socle de l'être et du sens du réel en tant que tel glisse de l'infini vers le fini. Cela ne signifie pas que toute évocation humaine de l'infini disparaisse. Au contraire, les modernes se donnent précisément pour tâche d'accéder à l'infini lui-même du seul point de vue qui devint admissible : celui de la facticité de la finitude humaine.

38. Dupré L., Passage to Modernity. An Essay in the Hermeneutics of Nature and Culture, New Haven, Yale University Press, 1993.

39. Ibid.

40. Foucault M., Sécurité, territoire, population, op.cit., p. 240.

41. Hunter I., Rival Enlightenments. Civil and Metaphysical Philosophy in Early Modern Germany, Cambridge, Cambridge University Press, 2002 ; Foucault M., Discipline and Punish. The Birth of the Prison, Harmondsworth, Penguin Books, 1982.

42. Dupré L., Passage to Modernity, op.cit. ; Blumenberg H., The Legitimacy of the Modern Age, Cambridge, Mass., MIT Press, 1983. 
Ainsi, cet infini que le projet de la modernité vise à accaparer - et à l'égard duquel il devient auto-enchanteur - partage-t-il certains de ses caractères avec l'éternité qui le précède à l'âge de la chrétienté, au point que certains, comme Schmitt, n'y voient que la sécularisation des catégories théologiques ${ }^{43}$. Il s'en distingue néanmoins très nettement. Le fini et l'infini sont comme un couplet. La ligne de démarcation des choses finies - on parlera de mortalité pour les êtres humains - ne peut manquer d'agir au-delà d'elle-même. Si le monde chrétien maintenait une division fondamentale entre l'éternité et la finitude des êtres mortels, posant dès lors le problème de l'articulation des deux, l'âge moderne inscrit l'éternel dans une temporalité, par l'immanentisation de l'eschaton. La fin des temps, la fin des choses, le jeu des dernières choses, devient un trope récurrent de la politique moderne, notamment dans les formes empiricistes biopolitiques. Plus on fuit la transcendance pour se réfugier dans le positivisme techno-scientifique, plus on devient conscient d'être hanté par un excès qui, toujours déjà en jeu dans le fonctionnement même des limites, menace en permanence la sécurité de ces limites positivement établies. Chercher à incorporer toujours plus l'au-dehors de ces limites, à la manière de la promesse manichéenne de la limite elle-même : la sécurité de la sécurité contre l'ennemi existentiel, la suppression de l'insurrection irrationnelle devant alors assurer la paix perpétuelle pour tous les êtres humains rationnels.

Pour les étudiants en relations internationales, ces deux processus étaient bien sûr à l'œuvre, in fine, dans le développement d'un système international interétatique et du droit international moderne. Un peu plus loin, dans la série Sécurité, territoire, population, Foucault donne une lecture assez traditionnelle de ces transformations et de la rationalité politique de la raison d'Etat qui leur est liée ${ }^{44}$. Toutefois, pour les étudiants intéressés aux questions de sécurité, il est aussi important de remarquer combien cette évolution historique complexe témoigne également du déclin progressif de la politique sotériologique de sécurité de l'Eglise, dont l'expression gouvernementale laïque de la communauté de croyance (ecumene), pour le dire en termes foucaldiens, était sa forme pastorale de gouvernement ${ }^{45}$. Dérivées d'une économie politique rédemptrice du salut ${ }^{46}$, la vie et la mort y sont figurées par rapport à la promesse de la vie éternelle. Lorsque, à la suite de ce que Foucault appelle des " insurrections de conduite » au XVie siècle ${ }^{47}$, la promesse de la vie éternelle perdit de sa force en tant que principe générateur de la formation du pouvoir gouvernemental de l'Eglise, les conditions matérielles de la vie comprise

43. Schmitt C., The Concept of the Political, op.cit., et Schmitt C., Political Theology II, op.cit.; Meier H., The Lesson of Carl Schmitt. Four Chapters on the Distinction between Political Theology and Political Philosophy, Chicago, Chicago University Press, 1998.

44. Voir Foucault M., Sécurité, territoire, population, op.cit., cours des 15, 22 et 29 mars 1978.

45. Ibid., cours 5-9.

46. Kantorowicz E. H., The King's Two Bodies. A Study in Mediaeval Political Theology, Princeton, Princeton University Press, 1982 ; Moore I., The Formation of a Persecuting Society, Princeton, Princeton University Press, 1990 ; Kaufman P. I., Redeeming Politics, Princeton, Princeton University Press, 1990.

47. Foucault M., Sécurité, territoire, population, op.cit., p. 233. 
comme vie finie, les conditions positives de possibilité - et non simplement la peur de la mort (qui pour Hobbes est, de façon paradigmatique, toujours entre les mains d'autres hommes) - ont progressivement acquis une signification $\mathrm{du}$ point de vue gouvernemental, en particulier au cours du XVII e siècle ${ }^{48}$.

Cela ne signifie pas qu'il faille ignorer l'argument très important de Kantorowicz dans Les deux corps $d u$ Roi ${ }^{49}$, où il retrace les origines de l'idée de perpétuité de l'Etat ( le roi ne meurt jamais ») dans l'édification de l'Eglise comme corps mystique qui, à son tour, ne peut jamais mourir. La manière dont le corpus mysticum de l'Eglise a donné naissance à l'idée de corps politique est tout à fait extraordinaire et regorge de paradoxes, à l'image d'ailleurs de l'idée ultérieure de perpétuité de l'Etat. Dans la politique moderne, comme dans la politique pré-moderne, la pensée de la finitude reste enveloppée par son homologue symétrique, l'infini. Il n'y a pas, en somme, de passage de l'une à l'autre comme s'il s'agissait d'un simple renversement binaire. L'enjeu est celui de la reformulation de tout l'ensemble qui découle d'une hiérarchisation et d'une reconfiguration de l'un sur l'autre ; l'apparition et la poursuite, pour ainsi dire, d'un principe différent de différenciation ${ }^{50}$. C'est la nature changeante du couplet fini/infini qui importe et explique l'intérêt qui est le mien pour les formes empirico-eschatologiques par lesquelles il se manifeste dans la modernité politique, dont la pensée de la finitude est également imprégnée par des expressions, des promesses et des espoirs eschatologiques. A cet égard, il est tout aussi important d'interroger les potentiels cultuels et théologiques des rationalités politiques et des technologies de gouvernement dans la modernité, à la manière de Walter Benjamin et de ses remarques cryptiques dans «Le capitalisme comme religion » 51 , que d'interroger les potentiels politiques de la re-ligio en tant que telle, sur les liens politiques qu'elle instaure : on pourrait retrouver cette question tout au long de l'histoire de la théologie en général et de la théologie politique en particulier, au moins en ce qui concerne la tradition chrétienne et post-chrétienne.

En revanche, nous pouvons dire que, alors que la politique sotériologique de sécurité de l’Église gouvernée par la promesse du salut était fondée sur une analytique de l'éternité, la politique moderne de sécurité en est venue à se

48. Hunter I., Rival Enlightenments. Civil and Metaphysical Philosophy in Early Modern Germany, Cambridge, Cambridge University Press, 2002 ; Kahn V., Wayward Contracts. The Crisis of Political Obligation in England 1640-1674, Princeton, Princeton University Press, 2004 ; Kahn V., Saccamano N., Coli D. (eds.), Politics and the Passions 1500-1850, Princeton, Princeton University Press, 2006 ; Oestreich G., Neostoicism and the Early Modern State, Cambridge, Cambridge University Press, 1982.

49. Kantorowicz E. H., The King's Two Bodies, op.cit.

50. Rotman B., Signifying Nothing. The Semiotics of Zero, Stanford, Stanford University Press, 1993 ; Rotman B., Ad Infinitum... The Ghost in Turing's Machine. Taking the God out of Mathematics and Putting the Body Back in, Stanford, Stanford University Press, 1993 ; Rotman B., Mathematics as Sign. Writing, Imagining, Counting, Stanford, Stanford University Press, 2000.

51. Benjamin W., “Capitalism as Religion”, in Bullock M., Jennings M. J. (eds.), Walter Benjamin Selected Writings, op.cit., pp. 288-291. 
baser sur une analytique de la finitude qui, malgré l'importance croissante accordée à la facticité de la finitude, a en grande partie imprégné l'ontothéologie pré-moderne eu égard, notamment, à la « subjectivité », la « raison » et même le rapport "salut/sécurité » 52 . Par conséquent, toute critique de notre politique moderne de sécurité - géopolitique et biopolitique de la sécurité incluses - toute critique de l'infinité de l'Etat par exemple, mais aussi de la finitude de l'homme, doit se tourner vers l'analytique de la finitude qui a été si déterminante pour sa production.

Une politique de sécurité basée sur la synthèse onto-théologique fracturée et limitée (finitised) - ayant gouverné l'analytique moderne de la finitude est relativement différente de celle basée sur la synthèse onto-théologique qui régissait autrefois l'analytique de l'éternité promue par l'Eglise. Il convient d'accorder plus d'attention à cette tâche, et en particulier à la façon dont les propriétés biologiques de l'être humain, comprises comme l'existence des espèces, sont devenues le point de référence pour asseoir des propriétés finies, le défaut empiriciste, vers lequel s'orientent les politiques modernes de sécurité tant par ses rationalités politiques que ses technologies de gouvernement. Un défaut qui, doit-il être précisé, code encore et toujours ses préoccupations eschatologiques propres et particulières. Peut-être l'ancien secrétaire américain à la Défense Donald Rumsfeld en a-t-il offert la meilleure expression dans ses remarques tristement célèbres à propos du «connu » et de "l'inconnu » qu'il conclût en affirmant que ce sont les « inconnus non connus » qui préoccupent désormais la politique de sécurité au XXIe siècle. Une remarque qui trouve son expression empirique dans l'obsession croissante des communautés de sécurité du bassin de l'Atlantique Nord pour la catastrophe, un événement défini comme catastrophique tant en vertu de son degré d'imprévisibilité - dépassant l'horizon du savoir rassurant, l'au-delà de la prévision normale, présageant de la fin du temps tel que nous le connaissons - que de sa puissance de dévastation matérielle.

Plus généralement, donc, le problème que Foucault a reconstitué historiquement à partir de cette littérature est celui du « comment être gouverné, par qui, jusqu'à quel point, à quelles fins et par quelles méthodes 53 ». Mettant en contraste cette littérature avec celle du Prince par exemple, Foucault observe que bien qu'elle fut majoritairement anti-machiavélienne, elle constitua aussi "un genre positif [avec] son objet, [...] ses concepts et [...] sa stratégie 54 ». Les travaux étudiés par Foucault couvrant un domaine des plus larges, à savoir le terrain entier de ce qui avait vocation à être gouverné et les diverses manières de le faire, la question du gouvernement de l'Etat trouva tout naturellement sa place dans ce large cadre de référence. Certains de ces travaux offraient une sorte de typologie des ontologies régionales du gouvernement,

52. Derrida J., On the Name, Stanford, Stanford University Press, 1995.

53. Foucault M., Sécurité, territoire, population, op.cit., p. 92.

54. Ibid., p. 94. 
chacune caractérisée par les propriétés distinctives du domaine à gouverner en question, chacune tombant sous le coup d'une science ou d'un mode de réflexion particulier ${ }^{55}$. Ainsi, le gouvernement de soi était considéré comme relevant du domaine de la moralité tandis que, suivant une tradition remontant à l'oikos grecque, l'art de gouverner la famille relevait lui de l'économie, et celui de gouverner l'Etat, de la politique ${ }^{56}$. De cette façon, la politique se distinguait comme n'ayant affaire ni entièrement avec la moralité, ni avec l'économie. Et pourtant, tous trois étaient corrélés puisque combinés dans l'Etat. Ces différents modes de gouvernement surgissant au sein d'une société et d'un Etat, se posait dès lors le problème de leurs relations dans la diversité même : «c'est à l'intérieur de l'Etat que le père de famille va gouverner sa famille, que le supérieur du couvent va gouverner son couvent, etc. ${ }^{57}$ » « $\mathrm{Il}$ y a donc [observe Foucault], à la fois pluralité des formes de gouvernement et immanence des pratiques de gouvernement par rapport à l'Etat, multiplicité et immanence de cette activité, qui l'opposent radicalement à la singularité transcendante du Prince de Machiavel. 58 ».

Il était ainsi entendu qu'il y avait continuité ascendante et descendante au sein de l'Etat entre différents domaines cibles du gouvernement : une « continuité ascendante en ce sens que celui qui veut pouvoir gouverner l'Etat doit d'abord savoir se gouverner lui-même; puis, à un autre niveau, gouverner sa famille, son bien, son domaine, et, finalement, il arrivera à gouverner l'Etat 59 ». De manière similaire, une continuité descendante était également postulée en ce sens que, «quand un Etat est bien gouverné, les pères de famille savent bien gouverner leur famille, leurs richesses, leurs biens, leur propriété, et les individus, aussi, se dirigent comme il faut 60 ». C'est ici en particulier qu'émergea l'art de ce qui sera connu et répandu en Europe continentale sous le nom de police ou Polizeiwissenschaft 61 . La police, sous cette acception, ne désigne pas l'officier ou le bras armé de la loi auquel réfère quasi exclusivement le terme dans le monde anglo-saxon. Foucault explique que « cette ligne descendante, qui fait retentir, jusque sur la conduite des individus ou la gestion des familles, le bon gouvernement de l'Etat, c'est ce qu'on commence à appeler à cette époque-là, précisément la "police” 62 ».

Cruciale dans cette préoccupation émergente des arts généraux du gouvernement était la famille, et la clé pour la famille était l'économie ${ }^{63}$. En fin de

55. Ibid., p. 96.

56. Ibid., p. 96-97.

57. Ibid., p. 96.

58. Ibid.

59. Ibid., p. 97.

60. Ibid.

61. Neocleous M., The Fabrication of Social Order. A Critical Theory of Police Power, Londres, Pluto Press, 2000.

62. Foucault M., Sécurité, territoire, population, op.cit., p. 97-98.

63. Ibid., p. 98. 
compte, « l'art du gouvernement, tel qu'il apparaît dans toute cette littérature, doit répondre essentiellement à cette question : comment introduire l'économie - c'est-à-dire la manière de gérer comme il faut les individus, les biens, les richesses, comme on peut le faire à l'intérieur d'une famille, comme peut le faire un bon père de famille qui sait diriger sa femme, ses enfants, ses domestiques, qui sait faire prospérer la fortune de sa famille, qui sait ménager pour elle les alliances qui conviennent -, comment introduire cette attention, cette méticulosité, ce type de rapport du père de famille à sa famille à l'intérieur de la gestion d'un Etat ? 64 ». C'est grâce à ce rôle pivot alors joué par la famille dans la science de la police, rôle par lequel la science de la police devait jouer pendant longtemps dans l'art de bien gouverner l'Etat, que cette économie s’implanta au cœur de la problématique du gouvernement. C’est pourquoi, quand Foucault répète que le principal problème du gouvernement sera l'introduction de l'économie dans la pratique politique ${ }^{65}$, nous ne pouvons présumer qu'il se réfère au gouvernement ou à l'économie en tant que catégories universelles, bien au contraire. Il réfère plutôt à la problématisation historique et spécifique de chacune, à leur extension dans la science, et en particulier dans la «science de la police ». Ce qui avait émergé comme les présupposés ontopolitiques, les rationalités politiques, les formes institutionnelles et les technologies gouvernementales de l'Europe médiévale céda la place à ce qui allait devoir être périodisé comme le moderne.

Ce moderne n'est toutefois pas encore l'art du gouvernement biopolitique mais simplement un gouvernement. Bien d'autres développements durent avoir lieu avant que le gouvernement ne se concentre autour des propriétés biologiques de l'humain (the buman way of being) et que l'«économie » déborde les confins de la « famille». Foucault expliqua comment dans la même série de cours (Sécurité, territoire, population). C’est en premier lieu le registre sémantique même de l'économie, ainsi que les appareils technologiques associés à l'éclosion du capitalisme commercial et, plus tard, aux débuts du capitalisme industriel, qui devaient subir d'importantes transformations. Dans cette modification du registre sémantique gisait aussi un changement dans la problématisation du gouvernement. Ici précisément, alors que l'art d'exercer le pouvoir sous forme économique en vient à prendre l'«économie » pour principal objet, le mot lui-même commence à signifier autre chose : "le mot économie, dit Foucault, désignait une forme de gouvernement au XVI e siècle [...] 66 ». Mais le développement de cet art de gouvernement était contraint d'une part par la préoccupation pour la problématique onto-théologique, massive et abstraite, de la souveraineté (associée de surcroît à sa concentration sur la mise en place juridique et bureaucratique des structures de l'Etat moderne) d'autre part par la restriction de l'économie au domaine étroit de la famille qui était « trop faible et trop peu substantiel »

64. Ibid.

65. Ibid.

66. Ibid., p. 99. 
pour supporter un essor plus important de l'idée et des pratiques de l'« économie » et même, en fait, de l'idée et des pratiques de « gouvernement ».

A partir du XVIII siècle, pourtant, selon Foucault, le mot économie « [...] désignera un niveau de réalité, un champ d'intervention pour le gouvernement, à travers une série de processus complexes, et [...] absolument capitaux pour notre histoire $67 »$. La distinction faite ici est certes concise mais cruciale : «voilà ce que c'est que gouverner et être gouverné 68 ». Foucault est si lapidaire sur ce point que la distinction peut ne pas être remarquée. Elle mérite toutefois d'être détaillée.

En d'autres termes, ce qui « gouverne » au moment de l'émergence de la problématique du gouvernement au crépuscule de la modernité, c'est l'économie comprise dans les termes de l'économie de gestion du foyer et de la famille. «Gouverner une famille, explique Foucault, [...] ce n'est pas essentiellement avoir pour fin de sauver les propriétés de la famille, c'est essentiellement avoir comme objectif, comme cible, les individus qui composent la famille, leur richesse, leur prospérité 69 ». Cela signifie aussi « tenir compte des évènements qui peuvent arriver : les morts, les naissances ; c’est tenir compte des choses que l'on peut faire, qui sont par exemple les alliances avec d'autres familles 70 ». C'était là la façon dont l'art de gouverner, en tant que pratiques méticuleuses touchant à l'économie de la famille, était conçu et appliqué en premier lieu. «L'essentiel, c'est donc ce complexe d'hommes et de choses ${ }^{71}$ ». Le territoire et la propriété étaient bien sûr concernés, mais ils n’étaient pas alors les référents uniques du gouvernement ainsi conçu et pratiqué. C'est bien l'ensemble des hommes et des choses qui l'était : « le territoire [et] la propriété n'en étant, en quelque sorte, qu'une variable 72 ». De plus, la loi régresse « ou plutôt, dans la perspective de ce que doit être le gouvernement, la loi n'est certainement pas l'instrument majeur 73 »; «Là encore [remarque Foucault], on retrouve le thème qui a couru pendant tout le XVII ${ }^{e}$ siècle et qui est manifestement explicite au XVIII ${ }^{\text {e }}$ siècle dans tous les textes des économistes et des physiocrates, quand ils expliquent que ce n'est certainement pas par la loi que l'on peut effectivement atteindre les fins du gouvernement ${ }^{74}$ ».

$\mathrm{Au} \mathrm{XVIII}$ e siècle, on postule toutefois que l'économie n'est pas quelque chose qui caractérise tellement la réalité de la famille, pour la constituer ellemême comme une réalité vécue indépendante (une ontologie régionale de plein droit) soumise à ses propres principes autonomes de formation, ses lois

\author{
67. Ibid. \\ 68. Ibid., p. 98. \\ 69. Ibid., p. 100. \\ 70. Ibid. \\ 71. Ibid. \\ 72. Ibid. \\ 73. Ibid., p. 103. \\ 74. Ibid.
}


et ses dynamiques propres, la meilleure façon de la gouverner étant posée comme problème de l'Etat. Et la réponse au dilemme du bon gouvernement, allait devoir se résumer dans la doctrine du laisser-faire. Un changement à noter. Dans le contexte du gouvernement des débuts de la modernité, c'est l'économie qui gouverne. Dans l'autre, celui connu rétrospectivement comme économie politique classique, "l'économie » est ce qui doit être gouverné, et gouverné au mieux en accord avec sa réalité propre et telle qu'elle s'était imposée.

Alors que le gouvernement en vint à être lié aux monarchies centralisées émergentes des XVII et XVIII ${ }^{\mathrm{e}}$ siècles et à leurs appareils administratifs en développement, il commença aussi à se fonder sur une forme de savoir connexe qui se répandait assidûment tant en Angleterre que sur l'Europe continentale ${ }^{75}$, ainsi que dans les colonies d'Amérique du Nord et plus tard, dans la République des Etats-Unis nouvellement indépendante ${ }^{76}$. Celui-ci était originellement connu sous le nom d'arithmétique politique. Autrefois réservé aux souverains et colonisateurs, ce mécanisme devint bientôt la science des statistiques, laissée à la disposition des progressistes et des réformateurs 77 . Une telle expertise fut alors associée, bien que de manière non exclusive, aux premiers jours du mercantilisme. Foucault nous dit ainsi que la première tentative visant à fournir une rationalité politique du pouvoir étatique était le mercantilisme. Le mercantilisme échouera par la suite, dit-il, " précisément parce qu'il s'[était] donné comme objectif [...] la puissance du souverain 78 ». Et ces instruments étaient ceux du souverain, « des lois, des ordonnances, des règlements, c'est-à-dire les armes même traditionnelles de la souveraineté 79 ». C'était comme si, explique Foucault, le mercantilisme ne pouvait pas développer plus avant les arts de gouverner car son horizon de pensée et de pratique était contraint par la façon dont il se concentrait sur la puissance du souverain plutôt que sur le développement des pouvoirs du gouvernement eux-mêmes.

75. Daston L., Classical Probability in the Enlightenment, Princeton, N.J., Princeton University Press, 1988 ; Hacking : Hacking I., The Taming of Chance, Cambridge, Cambridge University Press, 1990 ; Hacking I., The Emergence of Probability, Cambridge, Cambridge University Press, 1995.

76. Houston A., Population Politics: Benjamin Franklin and the Peopling of North America, Presented at the Center for Comparative Immigration Studies Research Seminar, San Diego, University of California, December 2003 ; Cassedy J. H., Demography in Early America. Beginnings of the Statistical Mind, 1600-1800, Cambridge, Mass., Harvard University Press, 1969.

77. Buck P., "Seventeenth-Century Political Arithmetic: Civil Strife and Vital Statistics", Isis, vol. 68, n 241, 1977, pp. 67-84 ; Buck P., "People who Counted in the Eighteenth Century", Isis, vol. 73, n 1, 1982, pp. 28-45; McLean S., The Event and its Terrors. Ireland, Famine and Modernity, Stanford, Stanford University Press, 2004 ; Hutchison T., The Collected Works of Sir William Petty, London, Routledge/Thoemmes, 1997; Phiddian R., "Political Arithmetick: Accounting for Irony in Swift's A Modest Proposal”, Accounting, Auditing, and Accountability Journal, vol.9, n5, 1996, pp. 71-83 ; Greer T. “Transmutation, Inclusion, and Exclusion: Political Arithmetic from Charles II to William III”, Journal of Historical Sociology, vol.20, n³, 2007, pp. 259-278.

78. Foucault M., Sécurité, territoire, population, op.cit., p. 105.

79. Ibid., p. 106. 
Par un paradoxe toujours aussi évident aujourd'hui, se concentrer sur les préoccupations du pouvoir souverain a pour effet de limiter son utilité pour la souveraineté. Comme Foucault le montre dans ses cours ${ }^{80}$, malgré ses préoccupations pour la métaphysique du pouvoir et les politiques de souveraineté territoriale, la souveraineté moderne est si minutieusement gouvernementalisée - préoccupée par tous les modes de régulation requis pour gouverner l'ensemble des hommes et des choses, d'autant plus lorsqu'elle proclame ses intentions limitées à leur égard - qu'elle a un appétit particulièrement insatiable pour cette inventivité en matière de développement de relations de pouvoir devant découler du maintien d'une distance critique par rapport à la souveraineté. Depuis le tout début de la souveraineté moderne, il est évident que les souverains ne pourraient pas satisfaire leurs ambitions de modernisation tout particulièrement celles relatives à l'institutionnalisation de la souveraineté elle-même - sans ce développement de relations de pouvoir dérivées du dehors des horizons onto-théologique et institutionnel de la souveraineté ellemême ${ }^{81}$. Si vous vous trouvez sur le marché des idées stratégiques à propos des relations de pouvoir, soyez certains que le souverain saura vous flairer. Mais le mercantilisme a essayé « de faire rentrer les possibilités données par un art réfléchi du gouvernement à l'intérieur d'une structure institutionnelle et mentale d'une souveraineté qui le bloquait 82 ». En ce qui concerne l'économie, aussi, le mercantilisme était confiné aux horizons d'une économie « qui, à cette époque-là encore, ne se référait jamais qu'à la gestion d'un petit ensemble constitué par la famille et la maisonnée 83 ».

La biopolitique apparaît donc, selon Foucault, comme le mouvement par lequel les contraintes des arts du gouvernement et de l'économie du début de la modernité furent levées par le glissement qui s'est opéré vers les propriétés biologiques de l'humain en général et vers le premier objet empirique de la gouvernance biopolitique - la population - en particulier. Une population est une multiplicité et non une communauté. Une population est une simple cohorte d'individus, qui, en masse (en italique dans le texte), affichent des propriétés observables et des traits caractéristiques. Ce sont eux qui sont devenus le nouvel objet, plus fertile, à la fois des arts du gouvernement et de l'économie, et ce, parce qu'ils sont devenus la cible d'un souci épistémique émergent centré sur les caractéristiques de l'existence de l'espèce. Puisqu'en effet l'économie était déjà installée au cœur du gouvernement, ce que Foucault décrit par la suite est un double processus parri passu : de développement de l'économie de gouvernement et de gouvernement de l'économie. Dès le milieu du XVIII siècle, en particulier en Angleterre et dans les colonies d'Amérique du Nord, au lieu de s'opposer aux instruments de l'arithmétique politique (recensements et statistiques) comme instruments de la tyrannie du pouvoir souve-

80. Ibid., p. 112.

81. Foucault M., Discipline and Punish, op.cit.

82. Foucault M., Sécurité, territoire, population, op.cit., p. 106.

83. Ibid., p. 107. 
rain, émancipateurs et réformateurs, les libres penseurs religieux aussi bien que les politiques adoptèrent les statistiques comme moyen de faire avancer leurs ambitions progressistes et émancipatrices ${ }^{84}$. Ils les utilisèrent comme moyen de pression, d'une part pour faire que l'ordre politique constitutionnel reflète les nouvelles classes et intérêts basés sur le niveau de richesse, d'autre part pour pousser le gouvernement à traiter des problèmes économiques et sociaux émergents 85 .

Comment donc, demande Foucault, l'art de gouverner fut-il libéré de ses contraintes ? Sa réponse générale établit une variété de facteurs parmi lesquels : "l'expansion démographique du XVIII ${ }^{e}$ siècle, reliée elle-même à l'abondance monétaire, reliée elle-même à l'augmentation de la production agricole 86 ». Mais bien sûr, bien d'autres éléments entraient en jeu. Son exposé n'est qu'une esquisse indicative, en aucun cas une histoire rigoureuse et exhaustive. Si tel avait été le cas, Foucault aurait pu poursuivre son observation à propos de l'augmentation des moyens financiers puisque ceux-ci aussi, là encore au sein de l'Empire britannique en particulier, étaient fonction de la dette publique 87 et de l'assurance ${ }^{88}$. En bref, la transformation de l'économie n'était pas simplement affaire de révolution des moyens de production. Pas plus d'ailleurs que ses effets n'étaient confinés à l'économie. Foucault s'est efforcé de retracer une transformation à l'œuvre au cœur des problématiques corrélées du gouvernement et de l'économie. En particulier, la révolution de l'économie impliquait aussi l'invention de nouveaux moyens financiers, et un plus grand accès au crédit, en particulier à la dette publique, qui était lui-même étroitement associé non seulement à l'expansion impériale et aux guerres pour l'hégémonie mondiale entre la France et l'Angleterre, mais aussi à l'essor conjoint de l'Etat-nation ${ }^{89}$.

A l'époque, tout comme aujourd'hui, l'importance de la dette publique était largement reconnue : par exemple et parmi d'autres, par David Hume. A l'époque, tout comme aujourd'hui, un grand scepticisme était exprimé à l'en-

84. Cassedy J. H., Demography in Early America. Beginnings of the Statistical Mind, 1600-1800, op.cit. ; Rusnock A. A., Vital Accounts. Quantifying Health and Population in Eighteenth Century England and France, Cambridge, Cambridge University Press, 2002.

85. Buck P., "Seventeenth-Century Political Arithmetic: Civil Strife and Vital Statistics”, op.cit. ; Buck P., "People who Counted in the Eighteenth Century", op.cit.

86. Foucault M., Sécurité, territoire, population, op.cit., p. 107.

87. Dickson P. G. M., The Financial Revolution in England, Londres, Macmillan, St Martin's Press, 1967 ; Poitras G., The Early History of Financial Economics, 1478-1776. From commercial arithmetic to life annuities and joint stocks, Cheltenham, Edward Elgar, 2000 ; Brantlinger P., Fictions of State - Culture and Credit in Britain 1694-1994, Ithaca, Cornell University Press, 1996.

88. Clark G., Betting on Lives. The Culture of Life Insurance in England 1695-1775, Manchester, Manchester University Press, 1999 ; Henndriks F., Contributions to the History of Insurance, and of the Theory of Life Contingencies, with a Restoration of the Gran Pensionary De Wit's Treatise on Life Annuities, Londres, C\&E Layton, 1851.

89. Brewer J., The Sinews of Power. War Money and the English State 1688-1783, Cambridge, Mass., Harvard University Press, 1988. 
contre de l'alchimie de l'argent, de la dette et du crédit. L'ambivalence de Defoe, exprimée au début du XVIII e siècle, était partagée par la plupart des lettrés et conservateurs de l'époque :

«Telle la lumière qui s’évanouit dans la nuit sombre, le crédit vient par surprise et disparaît sans qu'on le remarque... Lorsqu'il se répand sur une nation, il en double la force, il peut armer des flottes sans argent, avoir de l'argent dans fonds, et des fonds sans risque de défaut. Combien a accompli ce fantôme invisible pour cette nation, et combien de misères ont été subies auparavant sans lui ? 90 »

A l'époque, tout comme aujourd'hui, nonobstant les nombreuses différences que l'on peut établir entre les campagnes impériales mondiales du XVIII et $\mathrm{du} \mathrm{XXI}^{\mathrm{e}}$ siècle, la guerre en tant que vocation traditionnelle de la souveraineté ne pouvait pas être conduite sans les moyens financiers que l'invention de la dette publique et le développement de toutes sortes d'instruments financiers ont introduits au cours de cette période ${ }^{91}$.

Le développement, par exemple, d'une théorisation de l'endettement national et de la dette publique adéquate, quelque peu stabilisatrice, ou pour le moins stable, n'est apparu « qu'après l'éclatement de la bulle des Mers du Sud en 1721, et vraiment seulement dans la deuxième moitié du siècle, dans l'œuvre de David Hume, Adam Smith, Henry Thornton et autres 92 ». Marx aussi, bien sûr, reconnut que le mode capitaliste de production était basé sur la dette publique et la forme moderne de l'argent. En tant qu'expression du caractère plus large, à la fois significatif et troublant, de l'argent dans les jeux de hasard, et de risques introduits au cours du XVIII e siècle ${ }^{93}$, la finance publique en particulier (la dette publique) a été associée à la légitimité politique, au crédit national, et à ce que Hume appelait l'opinion. Comme Bratlinger, parmi d'autres, l'observe :

«Les Etats-nations belliqueux de l'Europe, dynamisés par l'expan-
sion au-delà de leurs frontières et l'industrialisation sur leurs terres,
étaient à même d'engager guerres et conquêtes impériales, et de s'in-
dustrialiser seulement sur la base de nouveaux arrangements fiscaux
généralisés. ${ }^{94}$ »

90. Cité dans Brantlinger P., Fictions of State, op.cit., p. 1.

91. Dickson P. G. M., The Financial Revolution in England, op.cit.; Brewer J., The Sineres of Power, op.cit. ; van der Heijden M., "State Formation and Urban Finances in Sixteenth and Seventeenth century Holland", Journal of Urban History, vol. 32, n 3, 2006, pp. 429-450; Ferguson N., The Ascent of Money, A Financial History of the World, Harmondsworth, Penguin Books, 2009.

92. Brantlinger P., Fictions of State, op.cit.; Brewer J., The Sinews of Power, op.cit.; Dickson P. G. M., The Financial Revolution in England, op.cit.

93. Simmel G., Philosophy of Money, Londres, Routledge, 1991 ; Rotman B., Signifying Nothing, op.cit. ; Shell M., Money, Language and Thought, Baltimore, The Johns Hopkins University Press, 1982 ; Ingham G., The Nature of Money, Cambridge, Polity Press, 2004. 
Et bien que ceci reste encore à être documenté en détail, la même chose peut être dite avec la même force à propos du phénomène de financement mondialisé de la dette et de la guerre impériale menée par les Etats-Unis et la Grande-Bretagne, en particulier au cours de la première décennie du XXIe siècle ${ }^{95}$. La biopolitique s'articule à la géopolitique au travers d'une large variété de mécanismes dont les troublants jeux de hasard associés à l'argent, la contingence et le risque ne sont pas les moindres.

Cela dit, Foucault s'est particulièrement intéressé à la façon dont la problématique de la population déplaça celle de la famille pour devenir le pivot autour duquel le gouvernement, l'économie, et les statistiques allaient aussi devoir se transformer. Le passage crucial ici est le suivant, et nécessite d'être cité en entier :

"Tout ceci étant le cadre général, on peut dire, de façon plus précise, que le déblocage de cet art de gouverner a été lié, je crois, à l'émergence du problème de la population. Ou disons encore, qu'on a un processus assez subtil, qu'il faudrait essayer de reconstituer en détail, dans lequel on verrait comment la science du gouvernement, le recentrement de l'économie sur autre chose que la famille et enfin, le problème de la population sont liés les uns aux autres. C'est à travers le développement de la science du gouvernement que l'économie a pu se recentrer sur un certain niveau de réalité que nous caractérisons maintenant comme économique, et c'est toujours à travers le développement de la science du gouvernement qu'on a pu découper le problème spécifique de la population. Mais on pourrait dire aussi bien que c'est grâce à la perception des problèmes spécifiques de la population et grâce à l'isolement de ce niveau de réalité qu'on appelle l'économie, que le problème du gouvernement a pu enfin être pensé, réfléchi et calculé hors du cadre juridique de la souveraineté. 96 »

De manière similaire, alors que le problème du gouvernement se reconcentrait sur les régularités quantifiables affichées par la population - tels que " son nombre de morts, son nombre de malades, ses régularités d'accidents 97 » les statistiques se détachaient aussi non seulement de la famille mais aussi de son identification antérieure à l'arithmétique politique des souverains ${ }^{98}$.

94. Brantlinger P., Fictions of State, op.cit., p. 22.

95. Ferguson N., The Ascent of Money, op.cit. ; Martin R., An Empire of Indifference. American War and the Financial Logic of Risk Management, Durham, Duke University Press, 2007.

96. Foucault M., Sécurité, territoire, population, op.cit., p. 107.

97. Ibid.

98. Buck P., "Seventeenth-Century Political Arithmetic: Civil Strife and Vital Statistics”, op.cit. ; Buck P., "People who Counted in the Eighteenth Century", op.cit.

99. Foucault M., Sécurité, territoire, population, op.cit., p. 107-108. 
«La statistique montre également que la population comporte des effets propres à son agrégation et que ces phénomènes sont irréductibles à ceux de la famille [...] : les grandes épidémies, les expansions endémiques, la spirale du travail et de la richesse. La statistique montre encore que, par ses déplacements, par ses manières de faire, par son activité, la population a des effets économiques spécifiques. [...] Sauf un certain nombre de thèmes résiduels, qui peuvent bien être des thèmes moraux et religieux, la famille comme modèle du gouvernement va disparaître. ${ }^{99}$ »

Ce que montre en fait l'analyse de Foucault est que ce qui commence à émerger n'est pas seulement l'essor de la population comme nouveau référent empirique stimulant à la fois les arts de gouverner et les sciences de l'économie et de la statistique - « la constitution d'un savoir de gouvernement est absolument indissociable de la constitution d'un savoir de tous les processus qui tournent autour de la population au sens large, ce qu'on appelle précisément l'“économie' 100 »- mais l'émergence et le développement au bout du compte d'une problématique plus large de gouvernement en référence aux propriétés biologiques de l'existence de l'espèce en général : le biopouvoir et la biopolitique.

Foucault est cependant très prudent lorsqu'il relève que « la souveraineté n’est absolument pas éliminée par l'émergence d'un art nouveau de gouverner, un art de gouverner qui a maintenant franchi le seuil d'une science politique. Le problème de la souveraineté n'est absolument pas éliminé ; au contraire, il est rendu plus aigu que jamais 101 ». Et une des manières par laquelle ce problème est rendu plus aigu encore relève précisément de sa relation aux nouvelles problématisations et aux arts de gouverner alors en essor. Ainsi, souligne-t-il : «[...] il faut bien comprendre les choses non pas du tout comme le remplacement d'une société de souveraineté par une société de discipline, puis d'une société de discipline, par une société, disons, de gouvernement. On a en fait un triangle : souveraineté, discipline et gestion gouvernementale dont la cible principale est la population et dont les mécanismes essentiels sont les dispositifs de sécurité 102 ».

Traversant ces trois éléments (souveraineté, discipline, et gouvernement) et contribuant matériellement à leur futur développement, la dépendance croissante à une pensée corrélative de la finitude s'affirmait alors. Mais, cette pensée de la finitude ne s'est pas révélée être un corps de pensée fixe et déterminé. La finitude n'est pas apparue non plus comme un point de référence aussi sûr et fiable pour la «vie » et sa gouvernance qu'on aurait pu le croire ou que certains le soutiennent. 